\title{
Estructura Factorial del Inventario de Trastornos de Ansiedad Generalizada en Universitarios Mexicanos
}

\author{
José R. Blanco, Martha Ornelas, Juan C. Barrón y Judith M. Rodríguez-Villalobos* \\ Universidad Autónoma de Chihuahua, Facultad de Ciencias de la Cultura Física, DES Salud CA 1121 , \\ Calle Escorza 900, CP 31000 Chihuahua, Chih.- México. (e-mail: jblanco@uach.mx, mornelas@uach.mx, \\ jcbarron@uach.mx, jurodrig@uach.mx)
}

*Autor a quien debe ser dirigida la correspondencia

Recibido Mar. 1, 2017; Aceptado May. 2, 2017; Versión final Jun. 20, 2017, Publicado Oct. 2017

\begin{abstract}
Resumen
El presente estudio analiza si es posible replicar los resultados psicométricos para el Inventario de Trastornos de Ansiedad Generalizada. La muestra total fue de 1307 estudiantes de las licenciaturas de Educación Física y Motricidad Humana que se ofrecen en la Universidad Autónoma de Chihuahua, con una edad media de 20.46 años. La estructura factorial del cuestionario se analizó a través de análisis factoriales confirmatorios. Los análisis, muestran que una estructura trifactorial es viable y adecuada. La estructura de tres factores (cognitiva, trastornos del sueño y síntomas somáticos), atendiendo a criterios estadísticos y sustantivos, ha mostrado adecuados indicadores de ajuste de fiabilidad y validez. Además, los resultados de los análisis factoriales llevados a cabo con las submuestras, indican la existencia de fuertes evidencias de la estabilidad de la estructura factorial. Futuras investigaciones deberían replicar estos hallazgos en muestras más amplias.
\end{abstract}

Palabras clave: ansiedad; estructura factorial; validación de constructo; ecuaciones estructurales

\section{Factor Structure of the Generalized Anxiety Disorder Inventory in Mexican University Students}

\begin{abstract}
The present study analyzes the possibility of duplicating the psychometric results for the Generalized Anxiety Disorder Inventory. The total sample comprised 1307 participants; all of them students of the degree of Physical Education and Human Motricity offered at the Autonomous University of Chihuahua, with a mean age of 20.46 years. Psychometric analysis showed that a Three -factorial structure was viable and adequate. The factorial structure of the questionnaire was analyzed with confirmatory factor analysis. The structure of the three factors (cognitive, sleep disorders and somatic symptoms), attending to substantive and statistic criteria, showed adequate indicators of adjustment of reliability and validity. In addition, factor analysis conducted with subsamples indicated the presence of strong evidence of factor structure stability. Future research should replicate these findings in larger samples.
\end{abstract}

Keywords: anxiety; factor structure; construct validation; structural equation 


\section{INTRODUCCIÓN}

Los desórdenes psicológicos son universales y parecen incrementar con el ritmo de vida actual. En América Latina y el Caribe los trastornos mentales con mayor prevalencia en personas mayores de 15 años fueron la depresión, la distimia, el estrés postraumático y la ansiedad generalizada según datos de la Organización Panamericana de la Salud (Rodríguez et al., 2009). Los jóvenes son un blanco vulnerable a este tipo de padecimientos debido a diversos factores socioeconómicos que se enfatizan mientras cursan la educación superior (Arrieta et al., 2014; Sanchez y Furlan, 2013). El ambiente universitario demanda en el estudiante grandes esfuerzos cognitivos y fisicos que promueven el desarrollo de estrés y ansiedad, ante situaciones académicas diversas entre las que destacan las evaluaciones, la sobrecarga extraclase y el tiempo limitado con que cuentan para cumplir con sus tareas (Nieves et al., 2013).

Específicamente en la población mexicana la ansiedad ha sido asociada al incremento en el consumo de drogas y alcohol en personas jóvenes, estos datos generaron que se prestara mayor atención a los programas de detección temprana de dichos trastornos (Caraveo et al., 2009). Por esta razón, es nuestro interés de estudio la atención oportuna del trastorno de ansiedad que a pesar de ser común en la población general, no es detectada ni atendida debidamente (Cárdenas et al., 2010). En América Latina más de la mitad de las personas que padecieron trastornos de ansiedad no fueron atendidas en un sistema de salud formal (Rodríguez et al., 2009). En este respecto se ha referido que existen múltiples barreras para el acceso a los servicios entre las que se menciona que los indicadores de salud mental se perciben como poco precisos (Kohn et al., 2005).

De los indicadores que se pueden abordar, el mejoramiento de las herramientas que ayuden a eficientar el diagnóstico es el principal punto de partida. Es evidente el requerimiento de instrumentos válidos y confiables que vayan más allá de conocer la prevalencia y permitan detectar de manera precisa los casos que presentan un nivel de ansiedad no óptimo. Si bien, hay instrumentos de evaluación y diagnóstico de la ansiedad al alcance de los profesionales de la psicología, todos tienen la limitante de que fueron diseñados bajo un contexto específico que no necesariamente se adaptan para todas las poblaciones de manera global.

Nos hemos enfocado en el Inventario de Desorden de Ansiedad Generalizada (GODI) por ser considerada una herramienta confiable con aceptable validez convergente y divergente que puede ser utilizada para el diagnóstico y ha sido sometida a validación con estudiantes universitarios (Spilios V Argyropoulos et al., 2007; Henderson et al., 2014), aun así es lógico tener en cuenta que nuestra población tiene necesidades particulares que posiblemente no correspondan a la situación propia de las personas con las que se logró el diseño original de dicho instrumento.

El presente estudio instrumental (Montero y León, 2005) se ha dirigido a proporcionar apoyo empírico a la división factorial del Inventario de Trastornos de Ansiedad Generalizada de Spilios V. Argyropoulos et al. (2007); lo que se justifica por la importancia de comprobar la estructura factorial de un instrumento y la equivalencia psicométrica del mismo en distintos grupos; ya que en el contexto de la comparación intergrupal, es indispensable plantearse la necesidad de llevar a cabo la adaptación de un instrumento de medida psicológica que cumpla con todos los criterios de equivalencia, pero sobre todo plantearse si la misma estructura factorial es aplicable a distintos grupos de sujetos 0 , de modo más genérico, a distintas poblaciones (Abalo et al., 2006). Por tanto, este trabajo pretende, por una parte, indagar si se replican los resultados psicométricos propuestos por Argyropoulos y colaboradores para el Inventario de Trastornos de Ansiedad Generalizada y, por otra, ampliarlos. Para ello, en primer lugar se comprobará el grado de congruencia de la estructura factorial de dicho cuestionario obtenida en el presente estudio y la reportada por Spilios V. Argyropoulos et al. (2007). En segundo lugar, se calculará la invarianza factorial entre las submuestras del presente estudio.

\section{METODOLOGÍA}

Se describe en subsecciones separadas, los participantes, el instrumento usado en el estudio, el procedimiento aplicado y finalmente se explica análisis de datos

\section{Participantes}

La muestra de 1307 participantes 708 mujeres y 599 hombres se obtuvo mediante un muestreo por conveniencia, tratando de abarcar la representatividad de las diferentes licenciaturas que se ofrecen en la Facultad de Ciencias de la Cultura Física de la Universidad Autónoma de Chihuahua. La edad de los participantes fluctuó entre los 18 y 26 años, con una media de 20.46 y una desviación estándar de 1.87 años. 
La muestra fue aleatoriamente dividida en dos partes utilizando el Statistical Package for the Social Sciences (SPSS) en su versión 21.0; con el fin de realizar estudios paralelos que permitieran corroborar los resultados obtenidos (validación cruzada). La submuestra 1 quedo constituida por 656 participantes 308 hombres y 348 mujeres, las edades fluctúan entre los 18 y 26 años, con una media de 20.45 y una desviación estándar de 1.88 años. La submuestra 2 quedo compuesta por 651 participantes 291 hombres y 360 mujeres, las edades fluctúan entre los 18 y 26 años, con una media de 20.46 y una desviación estándar de 1.85 años.

\section{Instrumento}

Inventario de Trastornos de Ansiedad Generalizada (GADI) de Spilios V. Argyropoulos et al. (2007). Cuestionario tipo Likert que consta de 18 ítems que se agrupan en tres dimensiones o subescalas: cognitiva, trastornos del sueño y síntomas somáticos; donde el encuestado responde, en una escala de 0 a 5 su grado de acuerdo con cada uno de los aspectos propuestos, útil para la evaluar la presencia o ausencia del trastorno por ansiedad generalizada así como su intensidad. La confiabilidad evaluada mediante el alfa de acuerdo a Henderson et al. (2014) es de .94 para la subescala cognitiva, .84 para la subescala que evalúa los trastornos de sueño y .89 para la subescala de síntomas somáticos. Se eligió este tipo de cuestionario por ser fácil de aplicar; además, de proporcionar una buena base para una ordenación de los individuos en la característica que se mide (Raviolo et al., 2010). Para el estudio que se presenta se hicieron tres adaptaciones a la versión original de Argyropoulos et al. (2007):

Primera adaptación, en la versión original se puntúa con seis opciones de respuesta, en la versión utilizada en la presente investigación el sujeto elige entre 11 posibles respuestas. Conjugamos la escala original con nuestra versión para que quedara de la manera siguiente: nunca (0), casi nunca (1, 2 y 3), a veces $(4,5$ y $6)$, casi siempre (7, 8 y 9) y siempre (10). Esta primera adaptación se justifica porque los sujetos al ser estudiantes están acostumbrados a la escala de 0 a 10, ya que así han sido evaluados por el sistema educativo de nuestro país (México).

En la segunda adaptación los ítems del cuestionario original fueron traducidos al castellano independientemente por dos investigadores expertos en construcción y validación de cuestionarios y con un alto conocimiento del inglés. Ambas versiones del cuestionario fueron contrastadas y debatidas en una sesión de análisis pormenorizado de cada ítem, donde se acordó finalmente la versión traducida más correcta. Se tuvieron en cuenta la familiaridad en el lenguaje y los tecnicismos usados en el original, la complejidad general del ítem y su nivel de abstracción, los tiempos verbales, el formato y puntuación del ítem, y se respetó la literalidad cuando ésta no modificaba el significado ni las expresiones resultantes en español. Posteriormente se procedió a la retro-traducción (back-translation). Una traductora profesional de lengua inglesa nativa, con amplia experiencia en la traducción de artículos científicos, tradujo los ítems del cuestionario que previamente se habían redactado en español. La tercera adaptación consistió en aplicar el instrumento por medio de una computadora, esto con el fin de permitir el almacenamiento de los datos sin etapas previas de codificación, con una mayor precisión y rapidez.

\section{Procedimiento}

Se invitó a participar en el estudio a los alumnos de las licenciaturas que se ofrecen en la Facultad de Ciencias de la Cultura Física (FCCF) de la Universidad Autónoma de Chihuahua. Los que aceptaron participar firmaron la carta de aceptación correspondiente. Luego se aplicó el instrumento, antes descrito, por medio de una computadora personal (módulo administrador del instrumento del editor de escalas de ejecución típica), en una sesión de aproximadamente 30 minutos; en los laboratorios o centros de cómputo de la FCCF. Al inicio de cada sesión se hizo una pequeña introducción sobre la importancia de la investigación y de cómo acceder al instrumento. Se les solicitó la máxima sinceridad y se les garantizó la confidencialidad de los datos que se obtuvieran. Las instrucciones de cómo responder se encontraban en las primeras pantallas; antes del primer reactivo del instrumento. Al término de la sesión se les agradeció su participación. Una vez aplicado el instrumento se procedió a recopilar los resultados por medio del módulo generador de resultados del editor de escalas versión 2.0 (Blanco et al., 2013).

\section{Análisis de datos}

El primer paso del análisis de las propiedades psicométricas del cuestionario consistió en calcular la media, la desviaciones estándar, la asimetría, la curtosis y los índices de discriminación de cada ítem. Para luego eliminar de la escala aquellos que obtienen una curtosis o asimetría extremas, o un índice de discriminación por debajo de .30. Luego, se sometieron a comparación dos modelos de medida: el Modelo 1 (M3), modelo de tres factores acorde a la distribución original de los ítems dentro del cuestionario y el Modelo 2 (M3b), 
que responde a la estructura factorial del modelo anterior, eliminando los ítems que no fueron suficientemente bien explicados por ese modelo y/o obtuvieron un índice de discriminación bajo.

Para conducir los análisis factoriales confirmatorios se utilizó el software AMOS 21 (Arbuckle, 2012), las varianzas de los términos de error fueron especificados como parámetros libres, en cada variable latente (factor) se fijó uno de los coeficientes estructurales asociados a uno, para que su escala sea igual a la de una de las variables observables (ítems). El método de estimación empleado fue el de Máxima Verosimilitud; siguiendo la recomendación de Thompson (2004), en el sentido de que cuando se emplea análisis factorial confirmatorio se debe corroborar no sólo el ajuste de un modelo teórico sino que es recomendable comparar los índices de ajuste de varios modelos alternativos para seleccionar el mejor.

Para evaluar el ajuste del modelo se emplearon el estadístico Chi-cuadrado, el índice de bondad de ajuste (GFI) y el error cuadrático medio de aproximación (RMSEA) como medidas absolutas de ajuste. El índice de bondad ajustado (AGFI), el Índice Tucker-Lewis (TLI) y el índice de bondad de ajuste comparativo (CFI) como medidas de ajuste incremental. La razón de Chi-cuadrado sobre los grados de libertad (CMIN/GL) y el Criterio de Información de Akaike (AIC) como medidas de ajuste de parsimonia (Byrne, 2010; Gelabert et al., 2011). Posteriormente, siguiendo las recomendaciones de Abalo et al. (2006), se llevó a cabo un análisis de la invarianza factorial del cuestionario para las submuestras tomando como base el mejor modelo de medida obtenido en la etapa anterior. Por último se calculó la fiabilidad de cada una de las dimensiones, de los modelos de medida obtenidos en cada submuestra, a través del Coeficiente Alpha de Cronbach (Elosua y Zumbo, 2008; Nunnally y Bernstein, 1995) y del Coeficiente Omega (Revelle y Zinbarg, 2009; Sijtsma, 2009).

\section{RESULTADOS}

En correspondencia con los análisis llevados a cabo para estudiar las propiedades psicométricas del instrumento se presentan los resultados descriptivos, el análisis confirmatorio y de invarianza factorial.

\section{Análisis descriptivos e índices de discriminación}

En la Tabla 1 se resumen los resultados de los análisis descriptivos y los índices de discriminación (correlación elemento-total corregida) de cada uno de los 18 ítems del cuestionario en la muestra total. Las respuestas a todos los ítems reflejan unas puntuaciones medias que oscilan entre 1.25 y 4.28 , y la desviación estándar ofrece, en todos los casos, valores mayores a 2.10 (dentro de un rango de respuesta entre 0 y 10). Con excepción del reactivo Me inquieta tener temblores y sacudidas (10) todos los valores de asimetría y curtosis se encuentran dentro del rango \pm 2.5 ; por lo que se infiere que las variables se ajustan razonablemente a una distribución normal. En cuanto a los índices de discriminación todos los ítems discriminan satisfactoriamente, obteniendo índices de discriminación por encima de .45 (Brzoska y Razum, 2010).

Tabla 1: Análisis descriptivos e índices de discriminación de los ítems del cuestionario "Inventario de Trastornos de Ansiedad Generalizada". Muestra total. $M=$ Media, DE= Desviación estándar, AS= Asimetría, $\mathrm{CU}=$ Curtosis, $\mathrm{r}^{\mathrm{i}-\mathrm{total}}=$ índice de discriminación.

\begin{tabular}{|c|c|c|c|c|c|}
\hline Ítem & $M$ & $D E$ & $A S$ & $C U$ & $r_{\text {i-total }}$ \\
\hline Item 1 & 2.89 & 2.63 & 0.69 & -0.34 & 0.69 \\
\hline Item 2 & 4.28 & 2.69 & 0.04 & -0.72 & 0.52 \\
\hline Item 3 & 3.63 & 2.75 & 0.39 & -0.62 & 0.61 \\
\hline Item 4 & 3.68 & 2.87 & 0.33 & -0.82 & 0.46 \\
\hline Item 5 & 3.88 & 2.84 & 0.33 & -0.74 & 0.50 \\
\hline Item 6 & 1.97 & 2.38 & 1.12 & 0.42 & 0.69 \\
\hline Item 7 & 2.56 & 2.58 & 0.84 & -0.18 & 0.63 \\
\hline Item 8 & 1.46 & 2.49 & 1.76 & 2.22 & 0.68 \\
\hline Item 9 & 1.53 & 2.24 & 1.51 & 1.54 & 0.65 \\
\hline Item 10 & 1.25 & 2.12 & 1.93 & 3.28 & 0.67 \\
\hline Item 11 & 2.69 & 2.73 & 0.77 & -0.34 & 0.62 \\
\hline Item 12 & 2.46 & 2.59 & 0.90 & -0.03 & 0.67 \\
\hline Item 13 & 1.84 & 2.56 & 1.39 & 1.03 & 0.65 \\
\hline Item 14 & 2.79 & 2.72 & 0.84 & -0.15 & 0.60 \\
\hline Item 15 & 2.59 & 2.73 & 0.90 & -0.10 & 0.72 \\
\hline
\end{tabular}




\begin{tabular}{|l|l|l|l|l|l|}
\hline Item 16 & 1.75 & 2.43 & 1.35 & 0.93 & 0.70 \\
\hline Item 17 & 3.60 & 3.00 & 0.46 & -0.80 & 0.68 \\
\hline Item 18 & 2.66 & 2.55 & 0.77 & -0.22 & 0.71 \\
\hline
\end{tabular}




\section{Análisis factoriales confirmatorios}

Los resultados globales del análisis factorial confirmatorio en la submuestra 1 (GFI .927; RMSEA .062; CFI .943) y la submuestra 2 (GFI .908; RMSEA .071; CFI .930) para el modelo M3 que corresponde a la distribución original de los ítems dentro del Inventario de Trastornos de Ansiedad Generalizada, indican que el modelo de medición, en ambas submuestras, se puede considerar como aceptable aunque no óptimo (Tabla 2).

Tabla 2: Índices absolutos, incrementales y de parsimonia para los modelos generados. Submuestras 1 y 2 . GFI = índice de bondad de ajuste; $\mathrm{NFI}$ = índice de ajuste normado; $\mathrm{CFI}$ índice de ajuste comparativo; RMSEA = raíz del error medio; AIC = criterio de Información de Akaike de cada uno de los modelos puestos a prueba en la invarianza factorial.

\begin{tabular}{|c|c|c|c|c|c|c|c|c|c|}
\hline & \multicolumn{3}{|c|}{ Índices absolutos } & \multicolumn{1}{c|}{ Índices incrementales } & \multicolumn{2}{c|}{ Índices de parsimonia } \\
\hline Modelo & $\chi^{2}$ & GFI & RMSEA & AGFI & TLI & CFI & \multicolumn{2}{c|}{ CMIN/DF } & AIC \\
\hline \multicolumn{8}{|c|}{ Primera solución factorial (submuestra 1) } \\
\hline M3 & $467.710^{*}$ & 0.927 & 0.062 & 0.906 & 0.934 & 0.943 & & 3.543 & 545.710 \\
\hline M3b & $252.503^{*}$ & 0.951 & 0.056 & 0.929 & 0.957 & 0.966 & 3.042 & 326.503 \\
\hline Segunda solución factorial (submuestra 2) \\
\hline M3 & $564.865^{*}$ & 0.908 & 0.071 & 0.881 & 0.919 & 0.930 & 4.279 & 642.865 \\
\hline M3b & $276.917^{*}$ & 0.944 & 0.060 & 0.919 & 0.955 & 0.965 & 3.336 & 350.917 \\
\hline
\end{tabular}

El conjunto de los tres factores del modelo M3 explican, en ambas submuestras, aproximadamente el $60 \%$ de la varianza. Por otro lado seis de los 18 ítems, en ambas submuestras, saturan por debajo de .70 en su dimensión prevista (2 Me preocupo por los acontecimientos cotidianos, 3 Me resulta difícil relajarme, $4 \mathrm{Me}$ siento "al límite", 5 Me despierto por la noche, 7 Sufro de sequedad en la boca y 14 Me asustan con facilidad). Observándose además, intercorrelaciones moderadas entre los tres factores evidenciando una adecuada validez discriminante entre ellos.

Los resultados globales del análisis factorial confirmatorio en la primer (GFI .951; RMSEA .056; CFI .966) y segunda submuestra (GFI .944; RMSEA .060; CFI .965), del segundo modelo sometido a prueba (M3b) que corresponde a una estructura tridimensional del cuestionario sin los ítems de más baja saturación en cada uno de los factores, indican que este modelo de medición es mejor que el modelo anterior y que su ajuste es óptimo (Tabla 2). Los tres factores de este modelo explican en conjunto, en ambas submuestras aproximadamente el $65 \%$ de la varianza. Por otro lado de acuerdo a los resultados de la Tabla 3; solo tres de los 15 ítems, en ambas submuestras, saturan por debajo de .70 en su dimensión prevista. Observándose además, intercorrelaciones moderadas entre los tres factores evidenciando una adecuada validez discriminante entre ellos.

\section{Invarianza de la estructura factorial entre las submuestras}

Los índices de ajuste obtenidos (Tabla 4) permiten aceptar la equivalencia de los modelos de medida básicos entre las dos submuestras. Aunque el valor de Chi-cuadrado excede al exigido para aceptar la hipótesis de invarianza, los índices $\mathrm{GFI}=.947, \mathrm{CFI}=.965$, RMSEA=.041 y $\mathrm{AIC}=677.420$ contradicen esta conclusión lo que nos permite aceptar el modelo base de la invarianza (modelo sin restricciones).

Añadiendo al modelo base restricciones sobre las cargas factoriales caracterizamos la invarianza métrica. Los valores que se recogen en la tabla 4 permiten aceptar este nivel de invarianza. El índice de ajuste general (GFI .946) y el error cuadrático medio de aproximación (RMSEA .040) siguen aportando información convergente en esta dirección. Además, el criterio de información de Akaike (AIC 664.792) y el índice comparativo de Bentler (CFI .965) no sufren grandes variaciones respecto al modelo anterior. Haciendo uso del criterio para la evaluación de los modelos anidados propuesto por Cheung y Rensvold (2002), quiénes sugieren que si el cálculo de la diferencia de los CFI de ambos modelos anidados disminuye en .010 menos, se da por bueno el modelo restringido y por tanto el cumplimiento de la invarianza factorial; la diferencia entre CFIs obtenida permite aceptar el modelo de invarianza métrica. Podemos concluir hasta ahora que las cargas factoriales son equivalentes en las dos submuestras.

Una vez demostrada la invarianza métrica entre las submuestras, pasamos a evaluar la equivalencia entre interceptos (invarianza factorial fuerte). Los índices (Tabla 4) muestran un ajuste aceptable de este modelo, tanto evaluado de modo independiente como analizándolo respecto a su anidamiento con el modelo de invarianza métrica. La diferencia entre los índices comparativos de Bentler es de .001; el índice de ajuste general es .944 y el error cuadrático medio de aproximación es .040 Aceptada la invarianza fuerte, los dos modelos evaluados son equivalentes respecto a los coeficientes factoriales y a los interceptos. 
Tabla 3: Soluciones estandarizadas análisis factorial confirmatorio para el Modelo M3b. Submuestra 1 y 2 . Nota: F1 = cognitiva, F2 = trastornos del sueño, F3 = síntomas somáticos

\begin{tabular}{|c|c|c|c|c|c|c|}
\hline \multirow[b]{2}{*}{ Item } & \multicolumn{3}{|c|}{ Submuestra 1} & \multicolumn{3}{|c|}{ Submuestra 2} \\
\hline & $\mathrm{F} 1$ & $\mathrm{~F} 2$ & F3 & $\mathrm{F} 1$ & $\mathrm{~F} 2$ & F3 \\
\hline \multicolumn{7}{|l|}{ Pesos Factoriales } \\
\hline 1. Estoy ansioso la mayoría de los días & 0.75 & & & 0.78 & & \\
\hline 3. Me resulta difícil relajarme & 0.67 & & & 0.67 & & \\
\hline 15. Tengo dificultad para controlar mi ansiedad & 0.81 & & & 0.80 & & \\
\hline 17. Me preocupo excesivamente & 0.74 & & & 0.71 & & \\
\hline 18. Estoy irritable & 0.78 & & & 0.74 & & \\
\hline 5. Me despierto por la noche & & 0.62 & & & 0.65 & \\
\hline 11. Tengo dificultad para conciliar el sueño & & 0.80 & & & 0.85 & \\
\hline 6. Experimento sofocos o escalofríos & & & 0.75 & & & 0.76 \\
\hline 7. Sufro de sequedad en la boca & & & 0.67 & & & 0.66 \\
\hline 8. Temo perder el control, desmayarme o volverme loco & & & 0.73 & & & 0.74 \\
\hline 9. Sufro de mareos & & & 0.73 & & & 0.75 \\
\hline 10. Me inquieta tener temblores y sacudidas & & & 0.74 & & & 0.74 \\
\hline 12. Sufro por la tensión o dolor en los músculos & & & 0.64 & & & 0.76 \\
\hline 13. Me inquieta la dificultad para respirar & & & 0.73 & & & 0.73 \\
\hline 16. Me inquieta sentir hormigueos o entumecimiento & & & 0.78 & & & 0.76 \\
\hline \multicolumn{7}{|c|}{ Correlaciones Factoriales } \\
\hline $\mathrm{F} 1$ & - & & & - & & \\
\hline $\mathrm{F} 2$ & 0.73 & - & & 0.71 & - & \\
\hline F3 & 0.77 & 0.66 & - & 0.86 & 0.76 & - \\
\hline
\end{tabular}

Tabla 4: Índices de bondad de ajuste GFI = índice de bondad de ajuste; NFI = índice de ajuste normado; CFI= índice de ajuste comparativo; RMSEA = raíz del error medio; AIC = criterio de Información de Akaike de cada uno de los modelos puestos a prueba en la invarianza factorial.

\begin{tabular}{|l|c|c|c|c|c|c|c|}
\hline \multicolumn{1}{|c|}{ Modelo } & \multicolumn{7}{c|}{ Índice de Ajuste } \\
\hline & $\chi^{2}$ & gl & GFI & NFI & CFI & RMSEA & AIC \\
\hline Modelo sin restricciones & $529.420^{*}$ & 166 & 0.947 & 0.951 & 0.965 & 0.041 & 677.420 \\
\hline Invarianza métrica & $540.792^{*}$ & 178 & 0.946 & 0.950 & 0.965 & 0.040 & 664.792 \\
\hline Invarianza factorial fuerte & $565.332^{*}$ & 184 & 0.944 & 0.947 & 0.964 & 0.040 & 677.332 \\
\hline
\end{tabular}

Los factores obtenidos en los análisis factoriales confirmatorios alcanzan, en su mayoría valores de consistencia interna por encima de .70 en ambas submuestras; evidenciando una consistencia interna adecuada para este tipo de subescalas, particularmente si se considera el número reducido de ítems (Tabla 5).

\section{DISCUSIÓN}

El objetivo principal del estudio fue indagar si se replican o no los resultados psicométricos propuestos por Spilios V. Argyropoulos et al. (2007) para el Inventario de Trastornos de Ansiedad Generalizada a través de una muestra de universitarios utilizando el análisis factorial confirmatorio (AFC). Los análisis factoriales confirmatorios realizados en cada submuestra por separado apoyan la estructura factorial de tres factores: cognitiva, trastornos del sueño y síntomas somáticos obtenida por Spilios V. Argyropoulos et al. (2007) al evidenciar una consistencia interna adecuada, particularmente si se considera el número reducido de ítems en cada uno de ellos; al mismo tiempo que los factores así obtenidos presentan en general saturaciones factoriales estandarizadas adecuadas, saturaciones que se corresponden con las halladas en el estudio de Spilios V. Argyropoulos et al. (2007). Sugiriendo además la existencia de fuertes evidencias de la validación cruzada de la medida y por tanto de la estabilidad de la estructura hasta que no se demuestre lo contrario. 
Tabla 5: Coeficiente omega y alfa para los factores obtenidos en los análisis factoriales exploratorios submuestras 1 y 2 .

\begin{tabular}{|l|c|c|c|c|}
\hline & \multicolumn{2}{|c|}{ Submuestra 1 } & \multicolumn{2}{c|}{ Submuestra 2 } \\
\hline \multicolumn{1}{|c|}{ Factor } & $\Omega$ & $\alpha$ & $\Omega$ & $\alpha$ \\
\hline Cognitiva & .865 & .867 & .859 & .863 \\
\hline Trastornos del sueño & .678 & .667 & .725 & .709 \\
\hline Síntomas somáticos & .896 & .896 & .905 & .906 \\
\hline
\end{tabular}

\section{CONCLUSIONES}

De acuerdo a lo presentado y discutido se puede concluir que el análisis de las propiedades psicométricas del Inventario de Trastornos de Ansiedad Generalizada, ha mostrado, tanto en este estudio como en el llevado a cabo por Spilios V. Argyropoulos et al. (2007), que la estructura trifactorial es viable y adecuada de acuerdo a los requisitos psicométricos establecidos cuando los informantes son los propios alumnos. La estructura de tres factores, atendiendo a criterios estadísticos y sustantivos, ha mostrado adecuados indicadores de ajuste, de fiabilidad y de validez.

Tomando en cuenta que el alcance de estos resultados es limitado, resulta necesario que:

1) En investigaciones futuras se confirme la estructura obtenida, lo cual permitirá contar con evidencia más robusta respecto a la estructura factorial de la escala. Específicamente, debe demostrarse si la invarianza de la estructura de la escala se cumple por género, edad, entre alumnos de distintas licenciatura.

2) Realizar estudios transculturales dirigidos a valorar si la estructura factorial de la escala es reflejo o no de diferencias culturales, de tal manera que, se considera que más estudios son necesarios con el fin de corroborar o refutar los datos obtenidos en las investigaciones realizadas hasta el momento.

3) Comprobar si la escala resulta útil para estudiar la relación entre la ansiedad y variables tales como el rendimiento académico y bienestar psicológico.

\section{REFERENCIAS}

Abalo J., J. Lévy, A. Rial y J. Varela, Invarianza factorial con muestras múltiples, En Modelización con Estructuras de Covarianzas en Ciencias Sociales por J. Lévy, 259-278. Netbiblo, Madrid, España (2006)

Arbuckle J. R., AMOS users guide version 21.0, Marketing Department. SPSS Incorporated, Chicago, USA (2012)

Argyropoulos S. V., G.B. Ploubidis y otros 9 autores, Development and validation of the Generalized Anxiety Disorder Inventory (GADI), doi: 10.1177/0269881107069944, J. Psychopharmacol, 21(2), 145-152 (2007)

Arrieta K. M., S. Díaz y F. González, Síntomas de depresión y ansiedad en jóvenes universitarios: prevalencia y factores relacionados, doi: 10.4321/s1699-695×2014000100003, Revista Clínica de Medicina de Familia, 7(1), 14-22 (2014)

Blanco H., M. Ornelas y otros 5 autores, Editor for creating and applying computerise surveys, doi: 10.1016/j.sbspro.2013.12.105, Procedia Soc. Behav. Sci., 106, 935-940 (2013)

Brzoska P. y O. Razum, Validity Issues in Quantitative Migrant Health Research: The Example of IIIness Perceptions, Peter Lang International Academic Publishers, New York, USA (2010)

Byrne B.M., Structural Equation Modeling With AMOS: Basic Concepts, Applications, and Programming, Routledge, New York, USA (2010)

Caraveo J., J. Rodríguez, R. Kohn y S. Aguilar-Gaxiola, Epidemiología psiquiátrica en México 1977-2000, En Epidemiología de los trastornos mentales en América Latina y el Caribe, 132-144, Organización Panamericana de la Salud, Washington, USA (2009)

Cárdenas E.M., M. Feria, L. Palacios y F. de la Peña, Guía clínica para los trastornos de ansiedad en niños $y$ adolescentes. En I. N. d. P. R. d. I. F. Muñiz, Ed., México (2010) 
Cheung G.W. y R.B. Rensvold, Evaluating goodness-of-fit indexes for testing measurement invariance, doi: 10.1207/s15328007SEM0902_5, Struct. Equ. Modeling; 9(2), 233-255 (2002)

Elosua P. y B.D. Zumbo, Coeficientes de fiabilidad para escalas de respuesta categórica ordenadas, Psicothema (en línea), 20(4), 896-901, (2008). https://goo.gl/AeyCe3. Acceso: Octubre (2016)

Gelabert E., L. García-Esteve, R. Martín-Santos, F. Gutiérrez, A. Torres y S. Subirà, Psychometric properties of the Spanish version of the Frost Multidimensional Perfectionism Scale in women, Psicothema (en línea), 23(1), 133-139, (2011). https://goo.gl/cvuZ1Q. Acceso Noviembre (2016)

Henderson L.C., M.M. Antony y N. Koerner, Psychometric properties of the Generalized Anxiety Disorder Inventory in a Canadian sample, doi: 10.1177/0269881114525672, J. Psychopharmacol, 28(5), 440-448 (2014)

Kohn R., I. Levav y otros 6 autores, Los trastornos mentales en América Latina y el Caribe: asunto prioritario para la salud pública, doi: 10.1590/S1020-49892005000900002, Revista Panamericana de Salud Pública, 18(4-5) (2005)

Montero I. y O. León, Sistema de clasificación del método en los informes de investigación en Psicología, Int. J. Clin Health Psychol., ISSN 1697-2600 (en línea), 5, 115-127 (2005). https://goo.gl/p1xRwk. Acceso Noviembre (2016)

Nieves Z.I., A. Satchimo, Y. González y J. Marisleydis, Algunas consideraciones acerca del estrés académico en los estudiantes universitarios. Some considerations about academic stress in college students, Psicoespacios, ISSN 2145-2776 (en línea), 7(11), 91-116, (2013). https://goo.gl/V1vQ5j. Acceso: Noviembre (2016)

Nunnally J.C. y I.H. Bernstein I. H., Teoría Psicométrica, McGraw-Hill, México (1995)

Raviolo A., P. Ramirez, E.A. López y A. Aguilar, Concepciones sobre el conocimiento y los modelos científicos: un estudio preliminar, doi: 10.4067/S0718-50062010000500005, Formación Universitaria, 3(5), 29-36 (2010)

Revelle W. y R.E. Zinbarg, Coefficients alpha, beta, omega and the glb: comments on Sijtsma, doi: 10.1007/s11336-008-9102-z, Psychometrika, 74(1), 145-154 (2009)

Rodríguez J.J., R. Kohn y S. Aguilar-Gaxiola, Epidemiología de los trastornos mentales en América Latina y el Caribe, PanAmerican Health Organization, Washington, USA (2009)

Sanchez J. y L. Furlan, Diferencias de género y situacional en universitarios, V Congreso Internacional de Investigación y Práctica Profesional en Psicología; XX Jornadas de Investigación; IX Encuentro de Investigadores en Psicología del MERCOSUR, Fac.de Psicología, Universidad de Buenos Aires (2013)

Sijtsma K., On the use, the misuse, and the very limited usefulness of Cronbach's alpha, doi: 10.1007/s11336-008-9101-0, Psychometrika, 74(1), 107-120 (2009)

Thompson B., Exploratory and Confirmatory Factor Analysis. Understanding concepts and applications. American Psychological Association, Washington, USA (2004) 